This item was submitted to Loughborough's Research Repository by the author.

Items in Figshare are protected by copyright, with all rights reserved, unless otherwise indicated.

\title{
Artificial situation awareness for increased autonomy of unmanned aerial systems in the terminal area
}

PLEASE CITE THE PUBLISHED VERSION

http://dx.doi.org/10.1007/s10846-012-9738-x

PUBLISHER

(C) Springer Verlag

VERSION

SMUR (Submitted Manuscript Under Review)

LICENCE

CC BY-NC-ND 4.0

REPOSITORY RECORD

McAree, Owen, and Wen-Hua Chen. 2012. "Artificial Situation Awareness for Increased Autonomy of Unmanned Aerial Systems in the Terminal Area”. figshare. https://hdl.handle.net/2134/11109. 
This item was submitted to Loughborough's Institutional Repository (https://dspace.lboro.ac.uk/) by the author and is made available under the following Creative Commons Licence conditions.

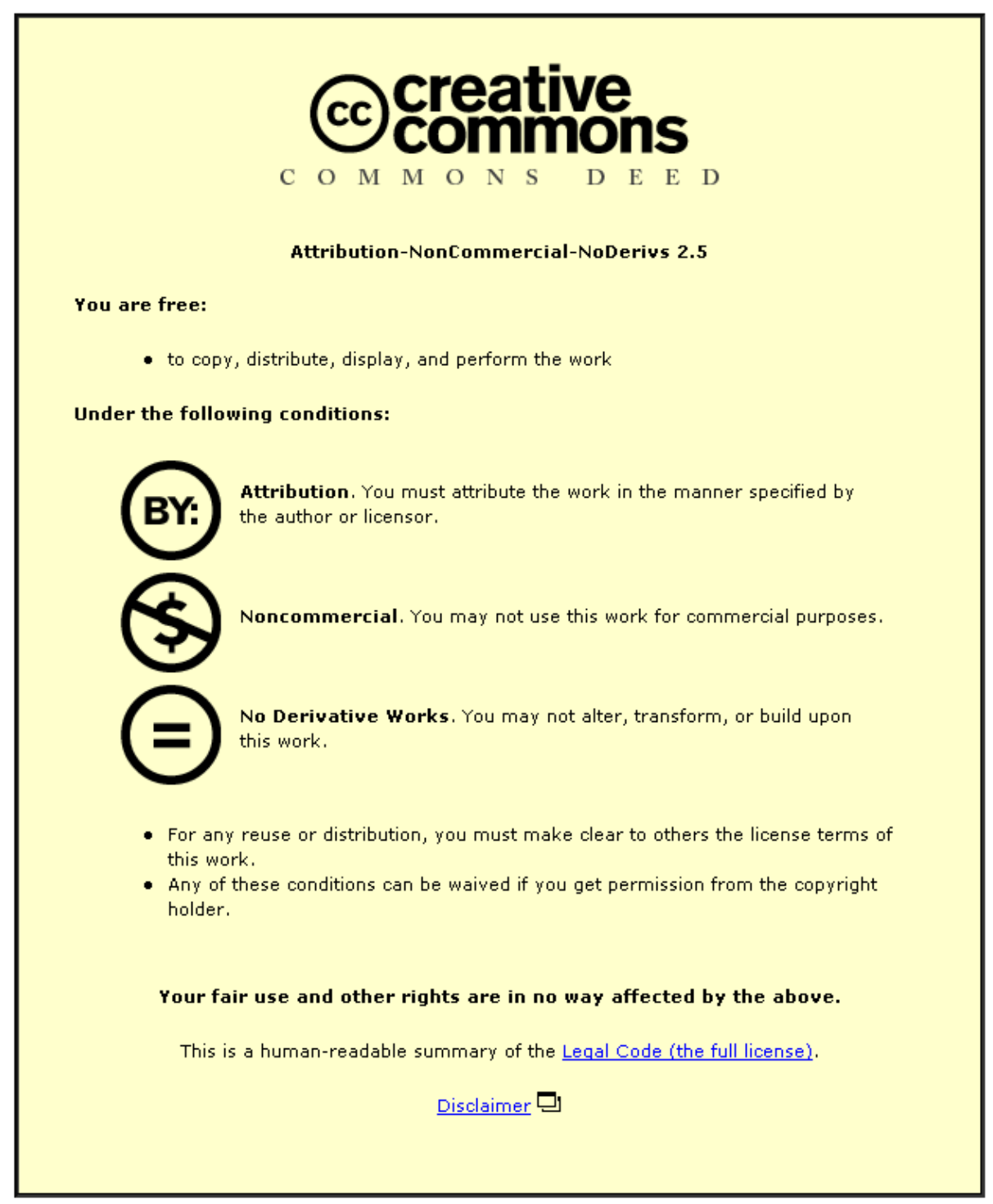

For the full text of this licence, please go to: http://creativecommons.org/licenses/by-nc-nd/2.5/ 
AUVSI11 - MCAREE

\title{
ARTIFICIAL SITUATION AWARENESS FOR AUTONOMOUS TERMINAL AREA OPERATIONS OF UNMANNED AERIAL SYSTEMS
}

\author{
Owen McAree; Wen-Hua Chen ${ }^{\dagger}$
}

\begin{abstract}
This paper provides an introduction to the development of artificial situation awareness for Unmanned Aerial System (UAS) operating close to a departure or arrival airfield (i.e. the terminal area). To construct artificial situation awareness a vehicle must be capable of perceiving and comprehending the current state of its environment (e.g. the positions of traffic), then predicting its future states. Focus will be on the comprehension and prediction elements because perception is assumed to occur via a broadcast system (such as Automated Dependant Surveillance Broadcast (ADS-B)). Comprehension includes both the current state of traffic in addition to its intentions, based upon the Rules of the Air. Knowledge of traffic intent allows a well refined prediction of future states to be made. Simulation results will be presented to demonstrate the benefits of artificial situation awareness with regards to safety and efficiency of UAS operations.
\end{abstract}

\section{INTRODUCTION}

Operation of Unmanned Aerial System (UAS) within civil airspace requires a number of challenges to be overcome to ensure equivalent levels of safety to manned operations. A major safety concern is the inevitable reduction in situation awareness of a UAS operator verses a manned aircraft pilot. This reduction is due to a number of factors including the loss of tactile and vestibular sensory information, the increased autonomy of the vehicle and the remote location of the operator. ${ }^{1,2,3}$ Situation awareness is most critical in highly dynamic environments the most important of which for UAS is the terminal area, the area immediately surrounding an airfield. Whilst mid-air collisions between manned aircraft are rare, the majority of occurrences are within the terminal area due to the convergence of vehicles to a single point in space. ${ }^{4}$ The reduction in situation awareness of a UAS operator within the terminal area would represent a significant reduction in safety thereby preventing the widespread integration of UAS with manned aircraft.

To mitigate the operators loss of situation awareness it is necessary to provide the UAS with a degree of artificial situation awareness to maintain a safe level of 'team' awareness. ${ }^{3}$ Whilst it is not anticipated that a UAS will operate completely independently of human supervision within the terminal area it is useful to consider this condition when discussing artificial situation awareness. This paper considers the requirement of an autonomous UAS to possess an equivalent level of situation awareness to a human pilot of a manned aircraft. As human oversight is always desirable

\footnotetext{
*Postgraduate researcher, Department of Aeronautical and Automotive Engineering, Loughborough University, Loughborough, UK. O.McAree@lboro.ac.uk

${ }^{\dagger}$ Senior Lecture, Department of Aeronautical and Automotive Engineering, Loughborough University, Loughborough, UK.W.Chen@lboro.ac.uk
} 
in a real world system the autonomy level of the UAS may be reduced in future to provide more control by the operator.

This paper focusses on one of the most important parts of terminal area situation awareness, that is the awareness of traffic vehicles. Other elements such as the internal state of the UAS and the weather are considered to be static and known absolutely by the UAS. A key element of this situation awareness is the projection of traffic positions in to the future and this is subject to a large degree of uncertainty. To capture these uncertainties a statistical approach to situation awareness has been used. Simulation results of the proposed artificial situation awareness methods are presented and further work such as the implementation on board a UAS are discussed.

\section{SITUATION AWARENESS}

When referring to a human operator, situation awareness is defined by Endsley as "The perception of the elements in the environment within a volume of time and space, the comprehension of their meaning and the projection of their status in the near future". 5 When considering a UAS in the terminal area the three tasks of perception, comprehension and projection can be broken down further to better describe the challenges.

\section{Perception}

Perception is more complex than simply sensing the environment as it involves the assignment of sensed data into meaningful units of information. Traffic in the terminal area must first be sensed either by cooperative means (e.g. Automated Dependant Surveillance - Broadcast (ADS-B)) or noncooperative means (e.g. radar). Non-cooperative sensing of traffic position may be either active (e.g. radar) or passive (e.g. electro-optical), both of which merely provide a UAS with information about the location of 'targets'. The UAS must assign each target a unique identifier in order to keep track of a particular vehicle as multiple measurements are taken over time. Additional data streams available to the UAS, such as Air Traffic Control (ATC) messages, may allow the UAS to determine the registration of the vehicle and use this as the identifier, a form of perceptual grouping.

Cooperative detection of traffic using ADS-B provides a unique datastream for each vehicle including the registration which enables straightforward grouping with ATC. For the purpose of this work the availability of ADS-B is assumed, simplifying the task of perception.

\section{Comprehension}

Comprehension is the task of making sense of the perceived information to build up an understanding of the environment. For a UAS manoeuvring in the terminal area comprehension of traffic is focused largely on building an understanding of the pilots intentions. In the first instance this is based largely on vehicle position based on a set of heuristics, e.g. "An aircraft in position X is likely to do Y'. Traffic vehicles may not always be considered independently at the comprehension phase as the relative positions of traffic may influence the decisions made by their respective pilots.

Additional refinement of comprehension may be performed by utilising ATC communication if, for example, a pilot announces their intentions or the controller issues an instruction. It is important, however, not to rely on this refinement as not all airfields operate a full ATC system. 


\section{Projection}

Projection is concerned with estimating the future states of the environment to enable more effective planning by the UAS. Traffic projection combines the tasks of perception and comprehension to estimate the future positions of vehicles. Perception provides information useful to short term projection, for example the current position and velocity of the vehicle enables the UAS to forecast its position for the next few seconds. Away from the terminal area it may be adequate to project forward the traffics position based on its current state for a longer time period. In the terminal area, however, this is not possible as this phase of flight contains a large number of manoeuvres conducted within a short space of time. Over a longer period of time, therefore, the vehicle intentions obtained from comprehension, must be used to predict its position. The terminal area presents a well defined problem for projection as typical flight paths are well defined.

\section{TRAFFIC COMPREHENSION}

The biggest single challenge a UAS faces within the terminal area is the convergence of a large amount of traffic to a small area of airspace. In practice traffic perception is a complex task if other vehicles do not possess some form of cooperative collision avoidance system (such as ADS-B). However, for the purpose of this work it is assumed that a means of traffic perception is already developed.

Based upon this perception of the current traffic state the UAS must process the information in order to generate a more meaningful state estimate, this is the task of traffic comprehension. The first stage in comprehension is to shift the state measurement to a local coordinate system and then estimate further continuous state variables in addition to discrete states. Continuous state variables are those such as velocities and accelerations, estimates of this information will allow more accurate projection to take place in the next stage. Discrete states are those such as circuit position or aircraft configuration and an estimate of these allows a better understanding of the traffics intentions.

\section{Local coordinate system}

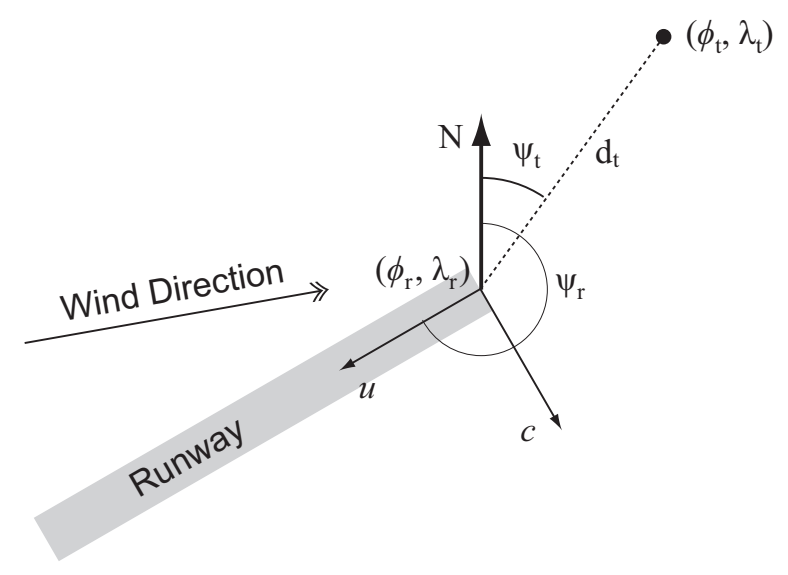

Figure 1. Coordinate System Transformation

Operation within the terminal area is done with reference to the active runway, determined by the current wind direction. The first comprehension task is to transform the positions of traffic 
from the Global Coordinate System (GCS) of latitude, longitude and altitude to a runway based Local Coordinate System (LCS), illustrated in Figure 1. The upwind axis $(u)$ is defined as along the centreline of the active runway in the direction of operation of that runway and crosswind $(c)$ is defined as pointing toward the 'live' side of the runway which is determined by circuit direction. Height is not shown in Figure 1 but is defined as positive up. The origin of the LCS is the runway threshold.

Transformation from GCS to LCS is a two stage process. First the position of the traffic $\left(\phi_{t}, \lambda_{t}\right)$ is transformed to a bearing and distance $\left(d_{t}, \psi_{t}\right)$ from the runway threshold $\left(\phi_{r}, \lambda_{r}\right)$, using the Haversine Formulae. This polar representation is then resolved into the Cartesian LCS and in addition to performing these translations for all traffic vehicles, the position of the UAS is also represented in the LCS.

\section{Hybrid state estimation}

Comprehension must include not only the continuous states such as velocity and acceleration but also discrete states such as the current circuit position of a vehicle. This is because ATC instruction is given with reference to circuit position and the traffics future intentions may be defined by its current position. Figure 2 illustrates these positions, or discrete states, in addition to a number of Flight Change Points (FCPs) which govern the transition from one state to the next. For example, FCP4 represents the point at which a vehicle transitions from the 'Late Downwind' state to the 'Turning on to Base' state. It is impossible to predefine these FCPs with any certainty as each vehicle will follow a different path. Additionally, the measurement of position is subject to error which will impact on the estimate of discrete states.

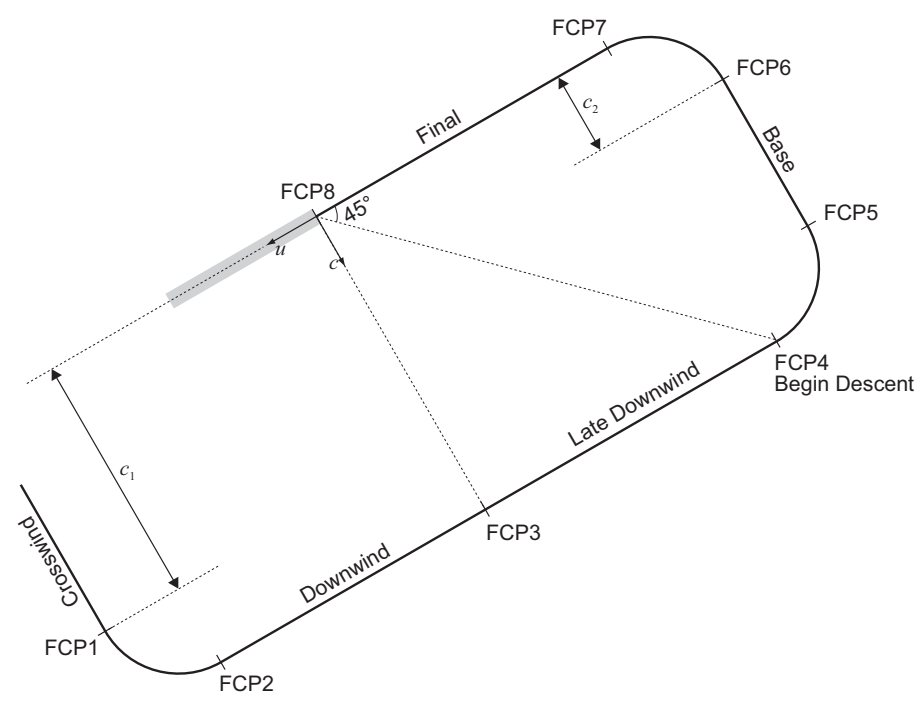

Figure 2. Discrete states within a circuit pattern

To mitigate the problems associated with uncertainty in FCPs and position measurement a State Dependent Transition Hybrid Estimation (SDTHE) algorithm can be used to both refine the continuous state estimation and more accurately assess the discrete states. ${ }^{6}$ The SDTHE algorithm breaks down each discrete state into lateral and vertical planes. In each state a vehicle may be in one of three lateral modes; Constant Velocity (CV), Constant Turn (CT) or Constant Acceleration (CA) and one of two vertical modes; Constant Height $(\mathrm{CH})$ or Constant Descent (CD). Referring to 
Figure 2, the transition at FCP4 can be characterised as a change from CV\&CH to CT\&CD as a vehicle turns toward the runway and begins its descent to land. Figure 3 illustrates the logic of the SDTHE. Each of the modes has an associated Kalman Filter (KF) which estimates the continuous states based on the assumptions made in that mode. The covariance matrices of each $\mathrm{KF}\left(P_{i}\right)$ are then used to determine the likelihood of the traffic being in that mode, the discrete state probabilities $(\alpha)$. Finally the KF continuous state estimates $\left(\hat{x}_{i}\right)$ are weighted by the discrete state probabilities to provide an overall continuous state estimate $(\hat{x})$.

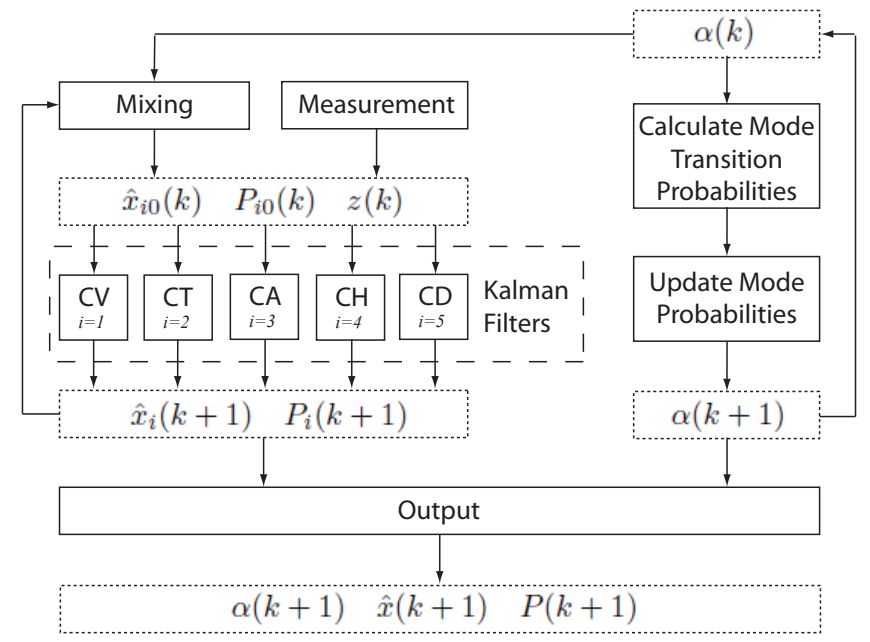

Figure 3. Execution logic of the SDTHE algorithm ${ }^{6}$

In order to test the robustness of the SDTHE to uncertainty in the location of the FCPs a number of tests were performed involving the estimation of a single vehicle, the results of one such test are illustrated in Figure 4.

\section{TRAFFIC PROJECTION}

The previous section discussed the comprehension of the current state, both continuous and discrete, of a traffic vehicle. This section extends the situation awareness of the UAS by performing projection of the traffic state to some future time horizon. Traditional collision avoidance algorithms perform a basic level of projection by assuming short term continuation of the currently observed state. When considering the terminal area, however, this short term projection is not sufficient for any more than emergency collision avoidance. This is due to the highly dynamic nature of the terminal area where traffic is unlikely to be maintaining a constant velocity for any length of time. To allow safe and efficient integration of UASs into terminal environments a long term projection system is needed which accounts for future discrete state transitions.

Projection may be abstracted into two distinct stages, short and long term. Short term projection assumes a continuation of the current state until the next FCP is reached. When the projected path reaches a FCP the current vehicle state can provide little information about the future path. At this point long term projection takes over by using a simple vehicle model to capture its dynamics and assuming the nominal circuit path will be flown. The assumptions about the dynamics and path of 

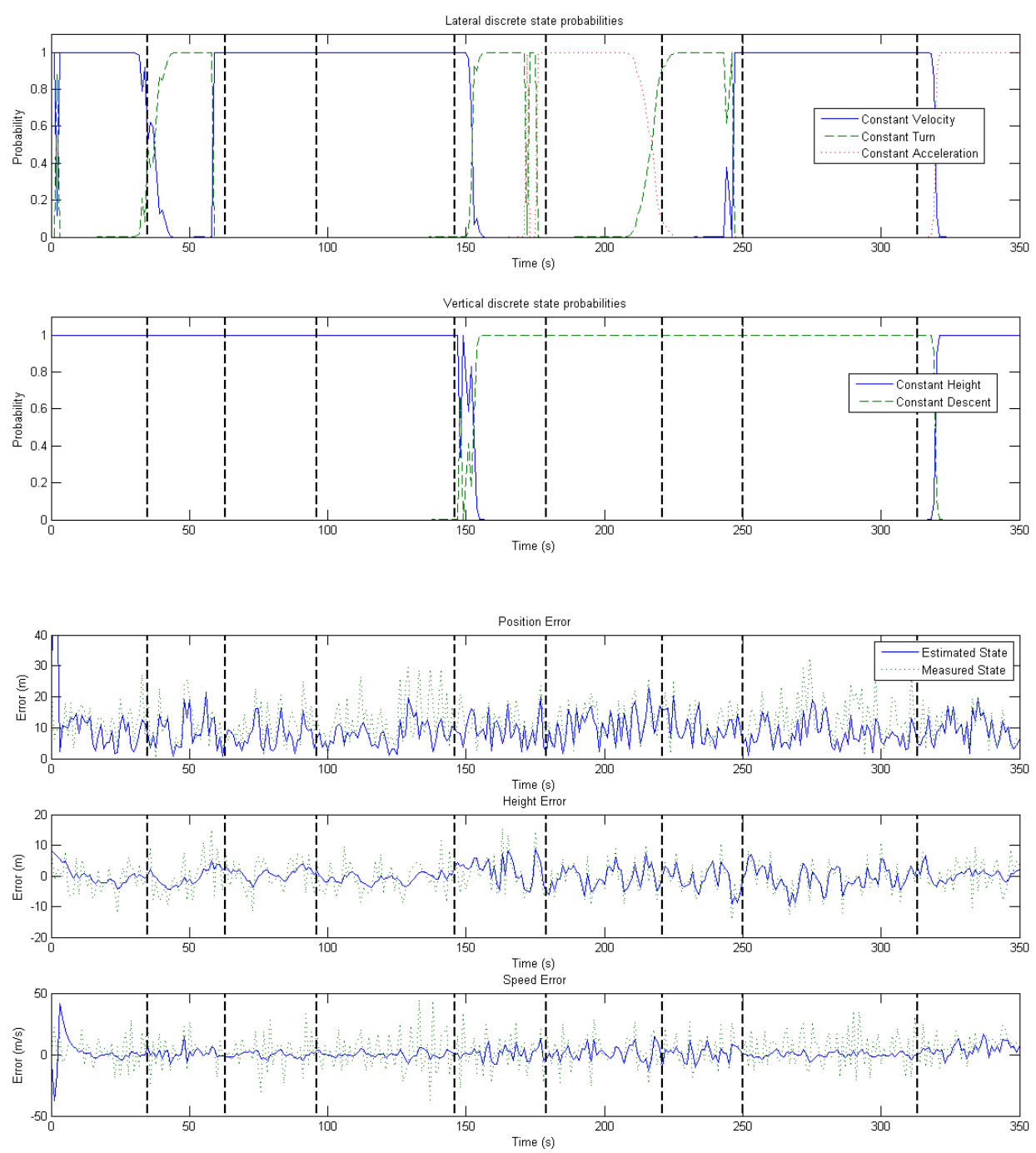

Figure 4. Plot of the discrete and continuous state estimation results

the vehicle are be subject to a large degree of uncertainty and quantification of this is a major part of the projection task.

\section{Positional Uncertainty}

When performing projection of a traffic vehicle assumptions about its flight path must be made based upon some nominal conditions. However, a vehicle may not fly the exact speeds and headings assumed by the UAS and will therefore deviate from the nominal projected path. To account for these uncertainties the position of the vehicle can be thought of as a Gaussian Multivariate Probability Density Function (MVPDF) with mean as defined by the nominal flight path and covariance defined by the uncertainties.

Uncertainties arising from the vehicles deviations from the nominal flight path are continuous in 
nature. They can be characterised as uncorrelated variances in the aircrafts velocity

$$
\Sigma_{v}=\left[\begin{array}{ccc}
\sigma_{v_{x}}^{2} & 0 & 0 \\
0 & \sigma_{v_{y}}^{2} & 0 \\
0 & 0 & \sigma_{\dot{h}}^{2}
\end{array}\right]
$$

where $\sigma_{v_{x}}^{2}, \sigma_{v_{y}}^{2}, \sigma_{\dot{h}}^{2}$ are the variances in velocity along the longitudinal, lateral and vertical aircraft axes respectively. Lateral velocity uncertainty may be difficult to define and can therefore be written in terms of a heading uncertainty

$$
\sigma_{v_{y}}=\hat{v} \tan \sigma_{\psi}
$$

where $\hat{v}$ is the nominal velocity and $\sigma_{\psi}$ is the variance in heading. This assumes that $\sigma_{v_{x}}$ is small in proportion to $\hat{v}$. Also for small values of $\sigma_{\psi}, \sigma_{v_{x}}=\sigma_{v}$, the velocity variance along the nominal flight path; and $\tan \sigma_{\psi}=\sigma_{\psi}$. Therefore Equation (1) may be written

$$
\Sigma_{v}=\left[\begin{array}{ccc}
\sigma_{v}^{2} & 0 & 0 \\
0 & \left(\hat{v} \sigma_{\psi}\right)^{2} & 0 \\
0 & 0 & \sigma_{\dot{h}}^{2}
\end{array}\right]
$$

To calculate positional uncertainty it is necessary to integrate the velocity uncertainties along the flight path and then transform into the LCS. It can be assumed that the velocity uncertainties remain constant throughout any given discrete state but they may change between states. Assuming the traffic is currently in discrete state $i$ and at projection time $t$ is nominally in discrete state $j$ the positional uncertainty, aligned with the aircraft axis is

$$
\Sigma^{\prime}(t)=\left[\begin{array}{ccc}
\sigma_{x}(t)^{2} & 0 & 0 \\
0 & \sigma_{y}(t)^{2} & 0 \\
0 & 0 & \sigma_{z}(t)^{2}
\end{array}\right]=\sum_{n=i}^{j} \Sigma_{v_{n}}\left(t_{n+1}-t_{n}\right)^{2}
$$

where $\Sigma_{v_{n}}$ is the $n$th discrete state velocity uncertainty, $t_{i}=0$ and $t_{j+1}=t$.

In order to transform Equation (4) into the LCS it is necessary to rotate the lateral and longitudinal terms by the heading of the vehicle (relative to the runway direction), $\psi^{\prime}(t)=\arctan \left(\frac{\dot{c}(t)}{\dot{u}(t)}\right)$. To rotate a MVPDF its covariance must be transformed by

$$
\Sigma(t)=R \Sigma^{\prime}(t) R^{T}
$$

where $R$ is the rotation matrix

$$
R=\left[\begin{array}{ccc}
\cos \psi^{\prime}(t) & -\sin \psi^{\prime}(t) & 0 \\
\sin \psi^{\prime}(t) & \cos \psi^{\prime}(t) & 0 \\
0 & 0 & 1
\end{array}\right]
$$

This transformation is analogous to multiplication by the square of the rotation matrix so as to maintain the correct dimensionality of $\Sigma$. Performing the transformation in Equation (5) yields

$$
\Sigma(t)=\left[\begin{array}{ccc}
\sigma_{u}(t)^{2} & \sigma_{u c}(t)^{2} & 0 \\
\sigma_{u c}(t)^{2} & \sigma_{c}(t)^{2} & 0 \\
0 & 0 & \sigma_{h}(t)^{2}
\end{array}\right]
$$




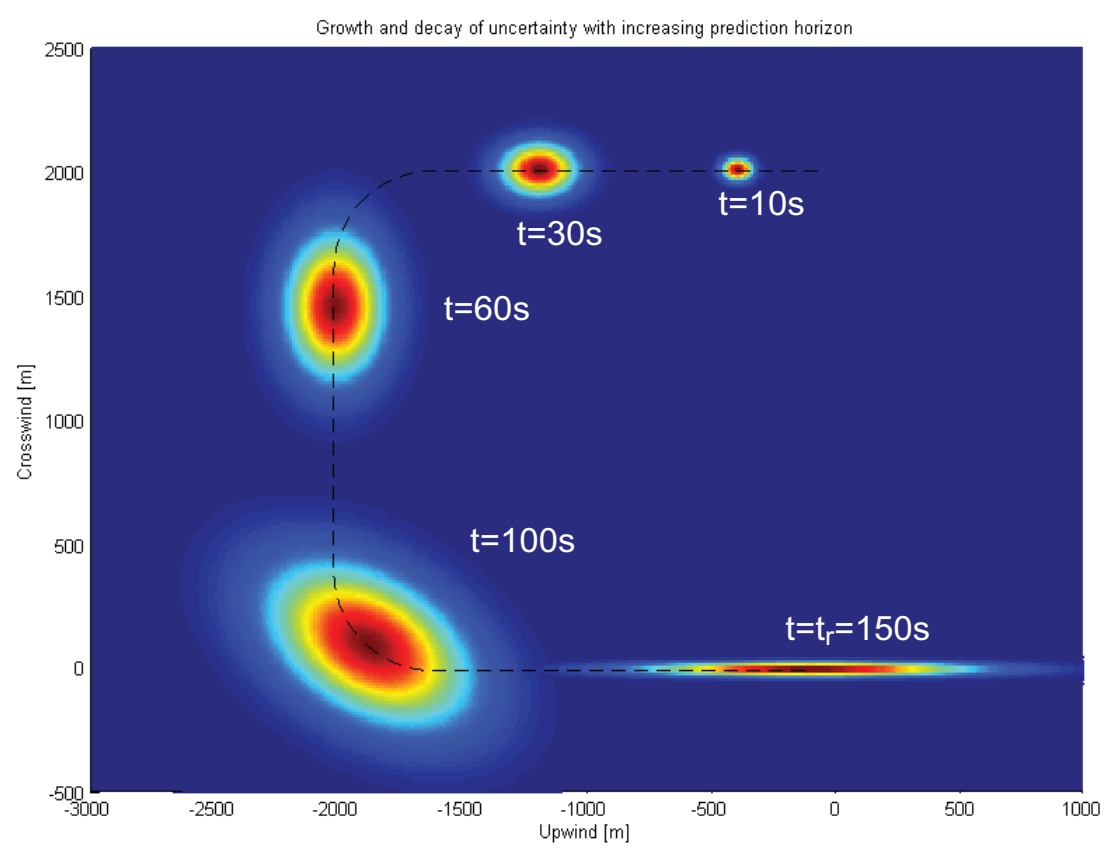

Figure 5. Growth and decay of uncertainty around a typical circuit

where $\sigma_{u}$ and $\sigma_{c}$ are the upwind and crosswind positional variances respectively and $\sigma_{u c}$ is their covariance. Given by

$$
\begin{gathered}
\sigma_{u}(t)^{2}=\left(\sigma_{x}(t) \cos \psi^{\prime}(t)\right)^{2}+\left(\sigma_{y}(t) \sin \psi^{\prime}(t)\right)^{2} \\
\sigma_{c}(t)^{2}=\left(\sigma_{x}(t) \sin \psi^{\prime}(t)\right)^{2}+\left(\sigma_{y}(t) \cos \psi^{\prime}(t)\right)^{2} \\
\sigma_{u c}(t)^{2}=\left(\sigma_{y}(t)^{2}-\sigma_{x}(t)^{2}\right) \sin \psi^{\prime}(t) \cos \psi^{\prime}(t)
\end{gathered}
$$

\section{Decay Of Crosswind Uncertainty}

It is clear that the positional uncertainty from Equation (7) will continue to grow as the prediction time grows due to the positive definite nature of $\Sigma_{v}^{\prime}$. The terminal area, however, presents a special case for projection and that is the eventual reduction in crosswind uncertainty. As prediction time grows the build up of uncertainty in velocity means that position can be forecast less and less accurately, however it is know that at some uncertain time in the future $t_{r}$ the vehicle will touchdown on the runway.

Figure 5 illustrates the growth and decay of uncertainty around a typical 2D circuit pattern. The (normalised) distributions at various projection times are shown and it can be seen that up to approximately $100 s$ the uncertainty grows as governed by Equation (7). Beyond $100 s$ however, uncertainty along the vehicles $x$-axis continues increasing, whilst along the $y$-axis it begins to decay. When the nominal projected position reaches the runway threshold the uncertainty has dropped to almost zero. It should be noted that this reduction can only practically occur in the last phase of flight when the pilot is aiming for the runway.

To achieve this reduction in uncertainty a simple exponential reduction in $\sigma_{y}$ is enforced from 
when the vehicle passes FCP7

$$
\Sigma_{\text {final }}^{\prime}(t)=\left[\begin{array}{ccc}
1 & 0 & 0 \\
0 & \left(\frac{\sigma_{y_{r}}}{\sigma_{y_{f}}}\right)^{\hat{v}_{f}\left(t-t_{f}\right) / 2 \hat{d}_{f}} & 0 \\
0 & 0 & 1
\end{array}\right] \Sigma^{\prime}(t)\left[\begin{array}{ccc}
1 & 0 & 0 \\
0 & \left(\frac{\sigma_{y_{r}}}{\sigma_{y_{f}}}\right)^{\hat{v}_{f}\left(t-t_{f}\right) / 2 \hat{d}_{f}} & 0 \\
0 & 0 & 1
\end{array}\right]^{T}
$$

where $\Sigma_{\text {final }}^{\prime}(t)$ is the (aircraft oriented) uncertainty covariance matrix for the final approach leg, $\sigma_{y_{f}}$ and $\sigma_{y_{r}}$ are the (aircraft oriented) lateral position uncertainties turning on to final approach and at the runway threshold respectively, $t_{f}$ is the projection time of passing FCP7 and entering the final leg, $\hat{v}_{f}$ is the nominal velocity of the final approach leg and $\hat{d}_{f}$ is the nominal distance of the final approach leg.

Equation (5) may then be used to translate the aircraft oriented uncertainties into the LCS.

\section{Temporal separation projection}

The previous section discussed the methodology for projecting forward in time the position of traffic based upon some knowledge of their current state and uncertainty in future states. If the future states of the vehicle were known with absolute certainty then calculation of the distance between the UAS and the traffic, and the subsequent transformation into the time domain, is trivial. This temporal separation information is required to allow a UAS to sequence itself within a circuit. It is undesirable, for example for a UAS to follow a manned aircraft too closely as there will be insufficient time for the manned aircraft to clear the runway prior to the UAS landing. Due to the uncertain nature of the traffics future state projection, however, the transformation to temporal separation is not straightforward.

Calculation of the separation between a pair of two-dimensional vectors is trivial if both are known precisely, however Figure 6a illustrates the problem if one point is described by a MVPDF. The position of the UAS is assumed to be known with absolute certainty and can be thought of as a point at a particular radius from the uncertain point. A probability distribution for the corresponding temporal separation exists as a function of the uncertainty of the traffic position but requires an uncorrelated distribution. ${ }^{7}$ To remove the correlation the problem must be transformed from an arbitrary point in space to a specific reference frame with the UAS as the origin and the axes aligned with those of the traffic, Figure 6b.

The probability distribution for separation is shown in Figure 7 for the following parameters.

$$
\begin{gathered}
{\left[\begin{array}{cc}
\sigma_{1}^{2} & 0 \\
0 & \sigma_{2}^{2}
\end{array}\right]=\left[\begin{array}{cc}
(500 m)^{2} & 0 \\
0 & (400 m)^{2}
\end{array}\right]} \\
{\left[\begin{array}{l}
\mu_{1} \\
\mu_{2}
\end{array}\right]=\left[\begin{array}{c}
600 m \\
-200 m
\end{array}\right]} \\
V_{U A S}=50 \mathrm{~ms}^{-1}
\end{gathered}
$$

Figure 7 also illustrates a Gaussian approximation utilising the Wilson-Hilferty transformation. $.8,9,10$ The approximation represents the distribution as standard (zero mean, unit variance) Gaussian distribution of the variable 


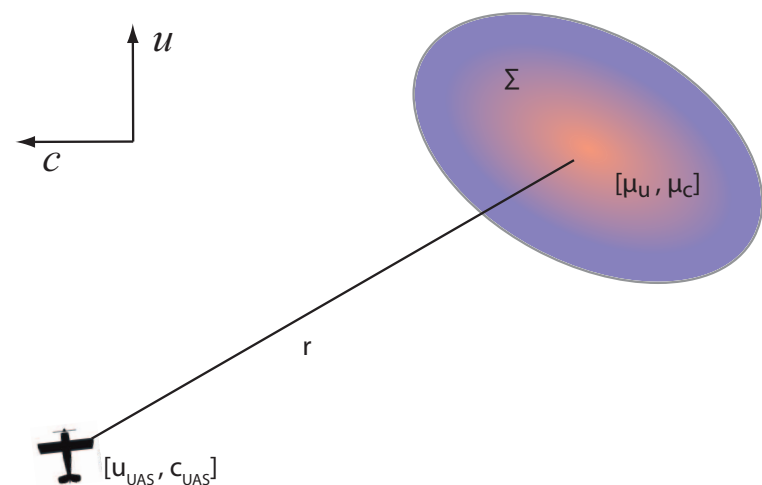

a)

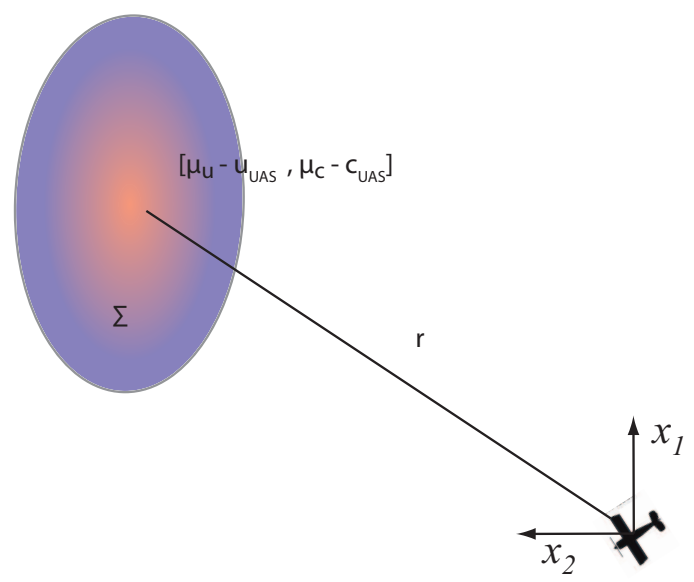

b)

Figure 6. Distance between and known and uncertain point. a) LCS b) transformed

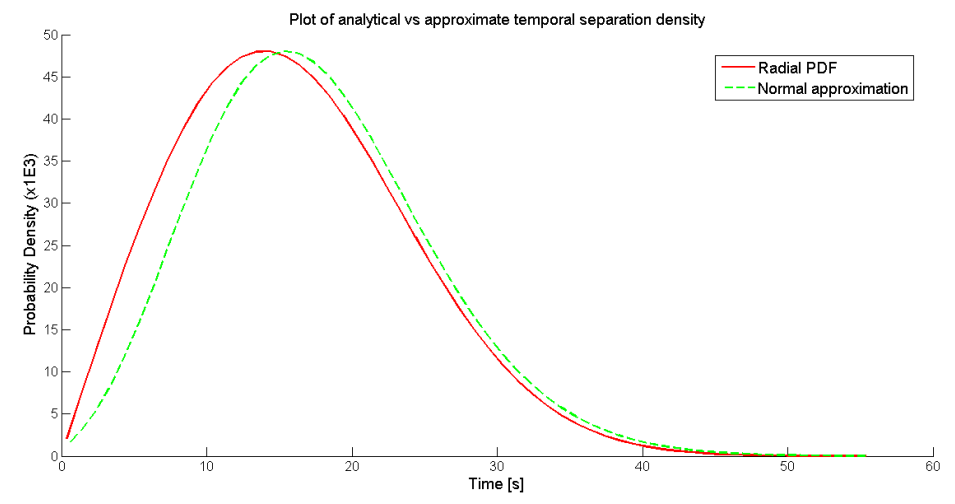

Figure 7. Comparison of analytical and approximate temporal distribution with comparative mean relative to variance

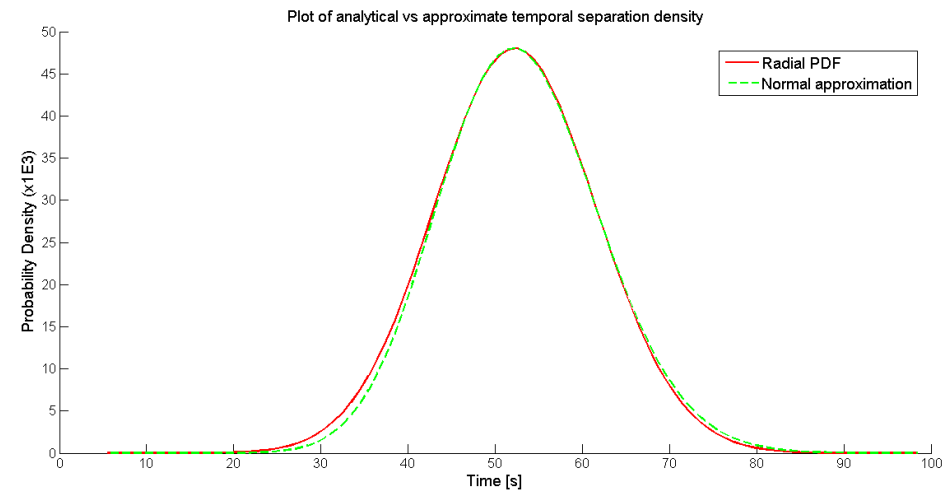

Figure 8. Comparison of analytical and approximate radial distribution with large mean relative to variance 


$$
p_{t}=\frac{\left(t^{2} /\left[\left(\sigma_{1}^{2} / V_{U A S}^{2}+\sigma_{2}^{2} / V_{U A S}^{2}\right) m\right]\right)^{\frac{1}{3}}-\left(1-v /\left[9 m^{2}\right]\right)}{\left(v /\left[9 m^{2}\right]\right)^{\frac{1}{2}}}
$$

where

$$
m=1+\frac{\mu_{1}^{2}+\mu_{2}^{2}}{\sigma_{1}^{2}+\sigma_{2}^{2}}
$$

and

$$
v=\frac{2\left(\sigma_{1}^{4}+\sigma_{2}^{4}+2 \sigma_{1}^{2} \mu_{1}^{2}+2 \sigma_{2}^{2} \mu_{2}^{2}\right)}{\left(\sigma_{1}^{2}+\sigma_{2}^{2}\right)^{2}}
$$

It can be seen that the shape of the approximate distribution is largely correct however it is skewed to the right. This discrepancy is caused by the highly non-Gaussian nature of the problem due to the reflection of the distribution about the origin. The problem arises when the variances $\left(\sigma_{1}\right.$ and $\left.\sigma_{2}\right)$ are of a similar size to the means $\mu_{1}$ and $\mu_{2}$ causing a large proportion of the distribution to appear behind the target point.

Figure 8 shows a comparison when the means are an order of magnitude greater than the variances $\left(\mu_{1}=2100 \mathrm{~m}\right.$ and $\left.\mu_{2}=1500 \mathrm{~m}\right)$. It is clear that the approximation is far better under these conditions as the analytical distribution tends closer to Gaussian.

It can be seen from Figure 7 that the reduction in accuracy encountered by the Gaussian approximation occurs when the separation time is much less than $30 \mathrm{~s}$. Whilst there is no predefined separation criteria between small aircraft with which UAS are likely to interact in the first instance (such as wake vortex separation between larger aircraft), a sufficient separation must be present to allow a leading vehicle to vacate the runway prior to the UAS attempting to land. It is proposed that when a UAS is making routing decisions based upon a prediction of the future state of traffic a temporal separation of at least $60 \mathrm{~s}$ is desired to account for errors in the prediction and allow sufficient time for runway vacation. Figure 8 illustrates that the performance of the Gaussian approximation at separation times closer to the $60 \mathrm{~s}$ target is excellent.

\section{Cumulative Temporal Separation}

The previous section looked at the transformation of the 2-dimensional uncertainty problem of traffic vehicle position to a single dimension uncertain time separation problem. The final task before a projection suitable for implementation within a planning system exists is to quantify this temporal separation problem by introducing a metric to measure separation. In a certain system this metric is simply the mean value, however in an uncertain system the Cumulative Distribution Function (CDF) is used to account for the variance. Mathematically the CDF is given as

$$
F\left(p_{t}\right)=\int_{-\infty}^{p_{t}} f(y) d y
$$

where $f(y)$ is a standard Gaussian Probability Density Function (PDF) and $p_{t}$ is the Gaussian approximation transformation, (10), for the time at which the CDF is to be evaluated.

Figure 9 shows how (11) can be used to measure separation between a UAS and traffic, it is produced using the same parameters as Figure 8. The curve represents the CDF and the intersection of the curve with $t=60 \mathrm{~s}$ is shown. The value of the CDF at $t=60 \mathrm{~s}$ is the probability of the UAS 


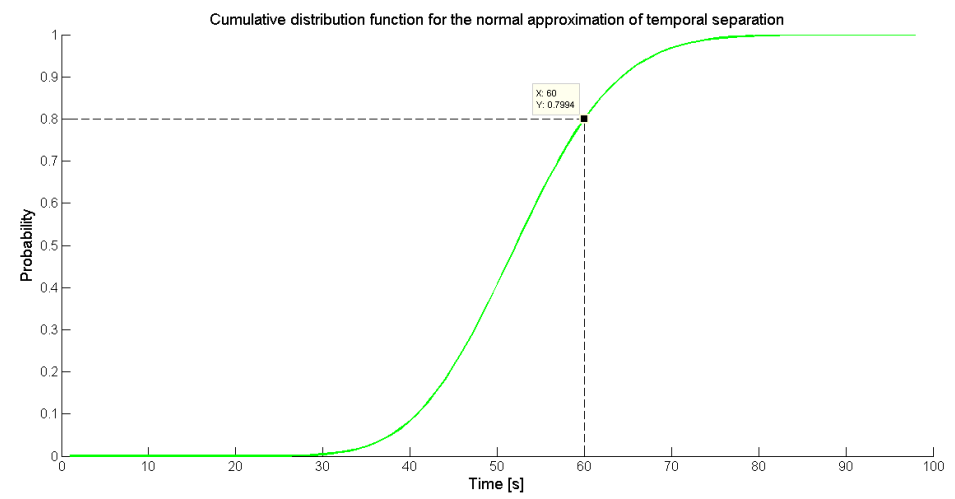

Figure 9. Cumulative distribution plot of temporal separation

being within $60 s$ of the traffic at the current projection step and this will be used as the Cumulative Temporal Separation (CTS). The CTS is therefore

$$
C T S=\int_{-\infty}^{p_{60}} f(y) d y
$$

\section{Temporal separation metric definition}

The Temporal Separation Metric (TSM) is defined as a measure of the separation between a UAS and a single traffic vehicle over the course of projection from the current point in time to some projection horizon $t_{p}$. It may be written as

$$
T S M_{v}=\frac{1}{t_{p_{v}}} \int_{0}^{t_{p_{v}}} C T S_{v}\left(X_{U A S}(t), X_{v}(t)\right) d t
$$

where the $v$ subscript refers to a specific traffic vehicle, $X_{U A S}(t)$ is the projected position of the UAS at time $t$ and $X_{v}(t)$ is the projected position of the traffic $v$ at time $t$.

The total TSM at any point in time is then simply the sum of all $n$ vehicles contributions, weighted to keep the parameter between zero and unity

$$
T S M=\frac{1}{n} \sum_{v=0}^{n} T S M_{v}
$$

The lack of subscript indicating the total value.

\section{TSM Example}

Figure 10 illustrates an example situation where the calculation of the TSM may benefit an autonomous UAS, although not specifically a terminal area scenario. Two traffic vehicles operating at $58 \mathrm{~ms}^{-1}$ and $46 \mathrm{~ms}^{-1}$ respectively are going to cross the path of the UAS, the radial separation of the vehicles is shown at intervals of $10 \mathrm{~s}$. Once per second the UAS is projecting forward both vehicles positions to a projection horizon of $60 \mathrm{~s}$ and by integrating their CTSs as discussed above the vehicles TSMs are found. These along with the total TSM are shown in Figure 11 for the entire $90 s$ simulation. 


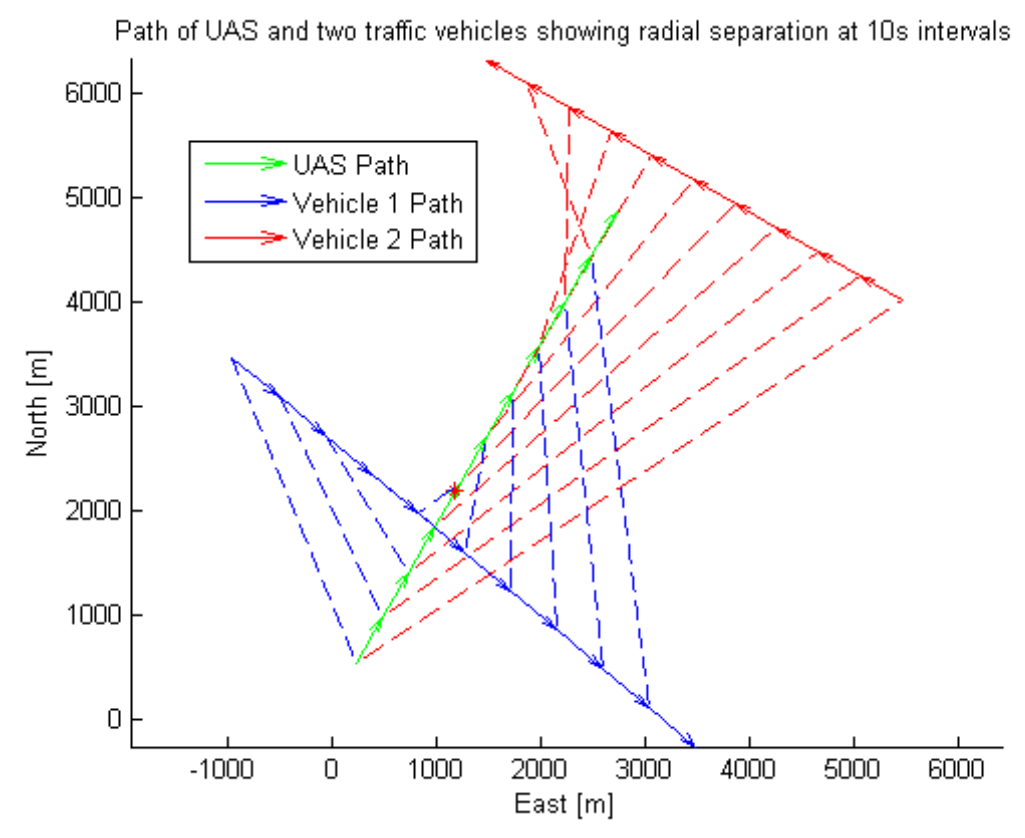

Figure 10. Simulation of UAS operating with two traffic vehicles

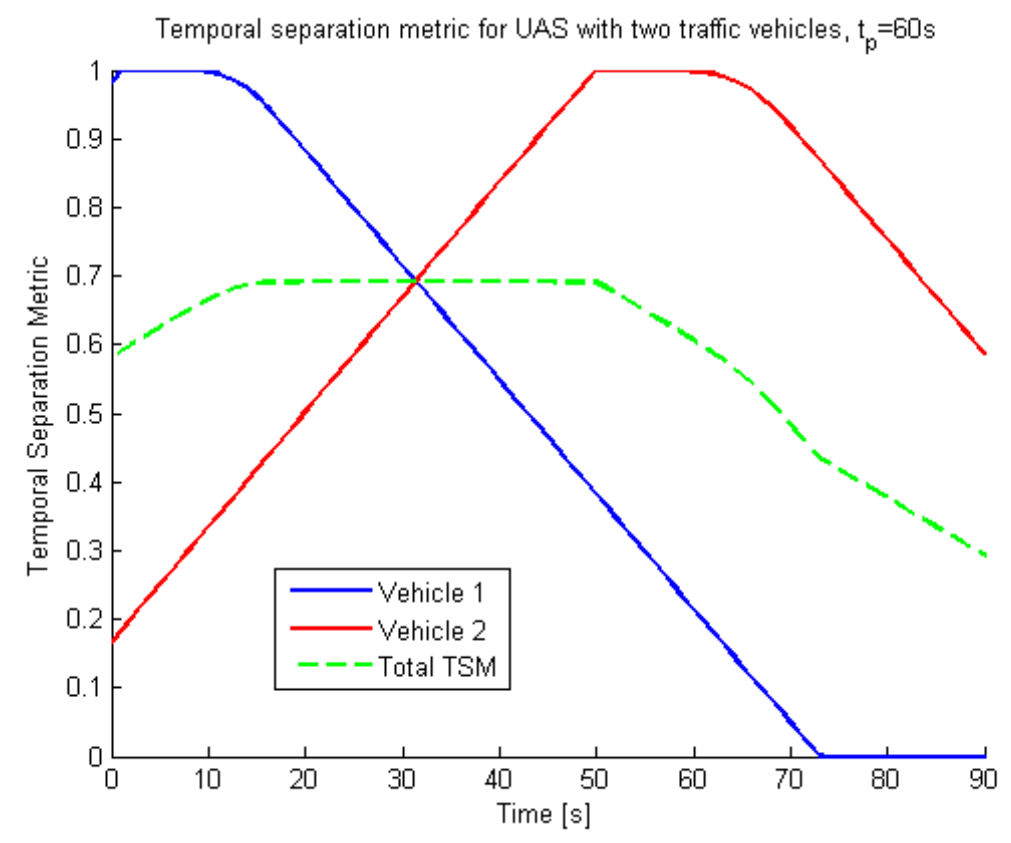

Figure 11. TSM calculated for traffic vehicles 
In this simulation the UAS is taking no action as a result of the computation of the TSM it is merely calculated to aid discussion. It may seem counterintuitive that after $10 \mathrm{~s}$ the TSM of vehicle $1\left(T S M_{1}\right)$ begins to reduce whilst it is still on a potentially conflicting course. This is due to the prediction horizon of $60 \mathrm{~s}$ looking beyond the potential conflict and seeing the temporal separation increasing. It is clear that an intelligent UAS making decisions on the basis of the TSM would take action within the first $10 \mathrm{~s}$ rather than simply continue on its current path so it is not believed that this behaviour is undesirable. However this behaviour does illustrate the importance of the selection of the correct $t_{p}$.

In a non-manoeuvring situation, for $t_{p} \leq 60 \mathrm{~s}$ an individual vehicles TSM will always saturate at unity when the separation time $t_{s}=60 s$ and $d t_{s} / d t<0$. This may be seen by the plot of $T S M_{2}$ in Figure 11 as it reaches unity at $t=53 \mathrm{~s}$ which is the point at which $t_{s}=60 \mathrm{~s}$. This it due to the entire projected path of the UAS being within the $60 \mathrm{~s}$ 'bubble' around the traffic. As the UAS continues to converge with the traffic and the temporal separation at the limit of projection $\left(t_{s}\right.$ at $t=t+t_{p}$ ) increases above $60 \mathrm{~s}$ the TSM will begin to reduce again as may be seen in Figure 11. The profile of this reduction is governed by the underlying uncertainty parameters describing the traffics projection.

After approximately $60 s$ both traffic vehicles have passed the UAS and the total TSM will continue to drop to zero. The red asterisk on the UASs path shows the point of minimum separation which occured at $t=38 \mathrm{~s}$ and was $t_{s}=9.2 \mathrm{~s}$. Clearly this is due to the lack of autonomy given to the UAS, however it should be noted that lack of autonomy closely resembles a 'pop-up' condition where the UAS is suddenly made aware of traffic in close proximity. If this had have been a pop-up condition the TSM would not have properly captured its urgency as figure 11 shows that $T S M_{1}(38 s)=58 \%$.

\section{CONCLUSION}

This paper has discussed the development of an artificial situation awareness system for autonomous UAS operating within the terminal area. The purpose of this work has been to mitigate the reduction in an operators situation awareness and maintain an equivalent level of safety to manned aircraft. It has been assumed that a system for perception of traffic vehicles is in place and on the basis of this a comprehension and projection method has been developed.

Comprehension has been tested for a single vehicle case and shows excellent performance in the estimation of continuous and discrete states. These state estimations form the basis of a projection system which is the main focus of this work. Projection forward of the positions of traffic vehicles accounting for the inherent uncertainties present in such an approach has created a system which an autonomous UAS may implement to make intelligent decisions regarding its integration to the terminal area.

\section{FUTURE WORK}

This work has produced a system which is capable of providing a UAS with a dynamic map of projected traffic positions up to some future time horizon. A UAS entering and operating within a terminal area is able to use this information to safely and efficiently plan its route and react to changes in the environment. Future work will focus on the implementation of the artificial situation awareness system within an intelligent path planning framework to allow autonomous integration of UAS in to the terminal area. 


\section{ACKNOWLEDGEMENT}

The authors would like to express their thanks for financial support for this project and the test facility from the Engineering and Physical Science Research Council (ERPSC), BAE Systems and UK Higher and Further Education Council. The assistance and support from other members in Autonomous Systems Laboratory at Loughborough are also greatly appreciated.

\section{REFERENCES}

[1] C. D. Wickens, "Situation Awareness and Workload in Aviation," Current Directions in Psychological Science, Vol. 11, 2002, pp. 128-133.

[2] C. D. Wickens, J. Lee, Y. D. Liu, and S. Gordon-Becker, Introduction to Human Factors Engineering (2nd Edition). Upper Saddle River, NJ, USA: Prentice-Hall, Inc., 2003.

[3] J. A. Adams, "Unmanned Vehicle Situation Awareness: A Path Forward," tech. rep., Vanderbilt University, 2007.

[4] NTSB, "Annual Review of Aircraft Accident Data U.S. General Aviation, Calendar Year 2003," tech. rep., NTSB, 2003.

[5] M. R. Endsley, "Design and evaluation for situation awareness enhancement," 1988.

[6] C. E. Seah and I. Hwang, "Terminal-Area Aircraft Tracking Using Hybrid Estimation," Journal of Guidance, Control, and Dynamics, Vol. 32, June 2009, pp. 836-849.

[7] H. Weil, "The Distribution of Radial Error," The Annals of Mathematical Statistics, Vol. 25, No. 1, 1954, pp. 158-170.

[8] E. B. Wilson and M. M. Hilferty, "The Distribution of Chi-square," Proceedings of The National Academy of Sciences, Vol. 17, 1931, pp. 684-688, 10.1073/pnas.17.12.684.

[9] F. E. Grubbs, "Approximate Circular and Noncircular Offset Probabilities of Hitting," Operations Research, Vol. 12, No. 1, 1964, pp. pp. 51-62.

[10] A. R. Eckler, "A Survey of Coverage Problems Associated with Point and Area Targets," Technometrics, Vol. 11, No. 3, 1969, pp. pp. 561-589. 